\title{
An exploratory factor analysis of nutritional biomarkers associated with major depression in pregnancy
}

\author{
Lisa M Bodnar ${ }^{1,2,3,4, *}$, Katherine L Wisner ${ }^{1,2,3,4,5}$, James $F$ Luther $^{1}$, \\ Robert W Powers ${ }^{3,4}$, Rhobert W Evans ${ }^{1}$, Marcia J Gallaher ${ }^{4}$ and PK Newby ${ }^{6}$ \\ 'Department of Epidemiology, University of Pittsburgh Graduate School of Public Health, A742 Crabtree Hall, \\ 130 DeSoto Street, Pittsburgh, PA 15261, USA: ${ }^{2}$ Department of Psychiatry, University of Pittsburgh School of \\ Medicine, Pittsburgh, PA, USA: ${ }^{3}$ Department of Obstetrics, Gynecology, and Reproductive Sciences, \\ University of Pittsburgh School of Medicine, Pittsburgh, PA, USA: ${ }^{4}$ Magee-Womens Research Institute, \\ Pittsburgh, PA, USA: ${ }^{5}$ Women's Behavioral HealthCARE, Pittsburgh, PA, USA: ${ }^{6}$ Departments of Pediatrics \\ and Epidemiology, Boston University Schools of Medicine and Public Health, Boston, MA, USA
}

Submitted 7 June 2011: Accepted 29 September 2011: First published online 8 December 2011

\begin{abstract}
Objective: Major depressive disorder (MDD) during pregnancy increases the risk of adverse maternal and infant outcomes. Maternal nutritional status may be a modifiable risk factor for antenatal depression. We evaluated the association between patterns in mid-pregnancy nutritional biomarkers and MDD.

Design: Prospective cohort study.

Setting: Pittsburgh, Pennsylvania, USA.

Subjects: Women who enrolled at $\leq 20$ weeks' gestation and had a diagnosis of MDD made with the Structured Clinical Interview for DSM-IV (Diagnostic and Statistical Manual of Mental Disorders, 4th edition) at 20-, 30- and 36-week study visits. A total of 135 women contributed 345 person-visits. Non-fasting blood drawn at enrolment was assayed for red cell essential fatty acids, plasma folate, homocysteine and ascorbic acid; serum 25-hydroxyvitamin D, retinol, vitamin E, carotenoids, ferritin and soluble transferrin receptors. Nutritional biomarkers were entered into principal components analysis.

Results: Three factors emerged: Factor 1, Essential Fatty Acids; Factor 2, Micronutrients; and Factor 3, Carotenoids. MDD was prevalent in $21.5 \%$ of women. In longitudinal multivariable logistic models, there was no association between the Essential Fatty Acids or Micronutrients pattern and MDD either before or after adjustment for employment, education or pre-pregnancy BMI. In unadjusted analysis, women with factor scores for Carotenoids in the middle and upper tertiles were $60 \%$ less likely than women in the bottom tertile to have MDD during pregnancy, but after adjustment for confounders the associations were no longer statistically significant.

Conclusions: While meaningful patterns were derived using nutritional biomarkers, significant associations with MDD were not observed in multivariable adjusted analyses. Larger, more diverse samples are needed to understand nutritiondepression relationships during pregnancy.
\end{abstract}

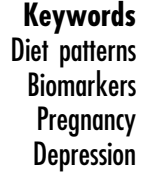

Keywords

Biomarkers

Depression
Depression is the leading cause of disease-related disability among women throughout the world, and the prevalence of depression is highest during the childbearing years ${ }^{(1)}$. One in seven women will develop depression during pregnancy or after birth ${ }^{(2)}$. Antenatal depression increases the risk of adverse outcomes, including preterm birth, pre-eclampsia, fetal growth restriction and altered neonatal and infant behaviour ${ }^{(3-5)}$. Pregnant depressed mothers are at risk of poor weight gain, substance abuse and failure to obtain adequate prenatal care ${ }^{(6)}$. Untreated major depression during pregnancy can lead to a worsening of the condition, thereby leading to suicide ideation or suicide attempts ${ }^{(7)}$ and a continuation of depression into the postpartum period ${ }^{(7)}$. Thus, prevention, identification and treatment of perinatal depression are major public health priorities.

Poor maternal nutritional status may be a modifiable risk factor for major depressive disorder (MDD). Nutrients are required for many aspects of brain functioning, including enzymatic activity, cellular and oxidative processes, receptor function, signal transmission, maintenance of neuronal tissue and synthesis and function of neurotransmitters 
and catecholamines. A wide range of nutrients has been linked with depression in adults ${ }^{(8-19)}$, but few investigators have studied nutrition and depression in the context of pregnancy ${ }^{(20-25)}$. Pregnant women are particularly vulnerable to nutritional deficiencies. Requirements for many key nutrients are higher during pregnancy than at any other time in the life cycle ${ }^{(26)}$. These needs are often difficult to meet with a typical Western diet or existing maternal stores. Moreover, standard prenatal vitamins may be inadequate for some nutrients (e.g. vitamin $\mathrm{D}$, essentially fatty acids). Identification of a relationship between maternal nutrition and MDD has the potential to lead to safe, inexpensive and acceptable interventions for prevention and treatment of mood disorders among pregnant women.

Our objectives in the present study were twofold. First, we used exploratory factor analysis to investigate whether there was a meaningful, underlying structure of maternal dietary biomarkers. Factor analysis is a powerful data reduction technique, but this approach has seen limited use in deriving patterns with nutritional biomarkers. Second, we evaluated the association between the dietary biomarker factors and MDD. We studied essential fatty acids, folate, homocysteine (Hcy), vitamins A, C, D and E, carotenoids and iron because these nutrients are of major public health interest and have been previously studied in relation to MDD or have a biologically plausible link to $\operatorname{MDD}^{(17,20,27)}$. Additionally, each nutrient has a biomarker of intake that is specific and highly predictive of the nutrient and represents integrated exposure over time ${ }^{(28)}$.

\section{Experimental methods}

The Antidepressant Use During Pregnancy (ADUP) Study is a prospective cohort study designed to examine the effects of maternal depression and antidepressant use on maternal and childhood outcomes ${ }^{(29)}$. Pregnant women with or without major depression and/or antidepressant use and singleton gestations were invited to enrol at $\leq 20$ weeks' gestation after providing informed, written consent. Study visits occurred at approximately 20, 30 and 36 weeks' gestation. At baseline, a non-fasting blood sample was obtained and data on sociodemographic characteristics, health behaviour and medication exposures were collected via interview. At each study visit, maternal depression was assessed by an experienced, trained clinician (details below). At the 30-week visit, usual dietary intake in the past 3 months was assessed with a modified Block98 FFQ. Approval was obtained from the University of Pittsburgh Institutional Review Board.

Women who were interviewed for study eligibility from April 2004 to January 2006 had blood samples processed for nutritional biomarkers. Of the 166 women interviewed during this period, $135(81 \%)$ provided a non-fasting blood sample at $\leq 20$ weeks that was processed for a full panel of nutritional biomarkers (described below). Of these, $73 \%$ had complete MDD diagnosis data at all three pregnancy study visits, while $16 \%$ and $11 \%$ had depression data available at two or one visit, respectively. Therefore, a total of 354 person-observations were included in the analyses.

At each visit, the diagnosis of major depressive disorder was made according to the Structured Clinical Interview for DSM-IV (Diagnostic and Statistical Manual of Mental Disorders, 4th edition) ${ }^{(30)}$ by an experienced, trained clinician. We adapted the timeline technique ${ }^{(31)}$ to chart major depression course by month across pregnancy ${ }^{(32)}$. Depression severity was assessed with the twenty-nineitem Structured Interview Guide for the Hamilton Rating Scale for Depression - Atypical Depression Symptoms Version $^{(33)}$, which includes all versions of the Hamilton Rating Scale for Depression ${ }^{(34)}$.

Fresh maternal blood was collected in red-top and purple-top (EDTA) tubes, which were kept in the dark and submerged in ice. Within $2 \mathrm{~h}$ of the blood draw, samples were centrifuged and stored in amber tubes at $-80^{\circ} \mathrm{C}$ until assay.

Fatty acids were extracted from red blood cells according to the general technique of Bligh and Dyer ${ }^{(35,36)}$. The CV were $5.5 \%$ for arachidonic acid (AA, $20: 4 n-6$ ), $4 \cdot 2 \%$ for EPA (20:5n-3) and $14 \cdot 4 \%$ for DHA (22: $6 n-3)$. Longitudinal control pools were run with each batch of assays. These showed excellent consistency.

Plasma folate was measured by a quantitative sandwich enzyme immunoassay technique on a 2010 Elecsys autoimmunoanalyser (Roche Diagnostics, Indianapolis, IN, USA). The CV was $2.0 \%$ to $3.9 \%$. The lowest detection limit of this assay is $0.6 \mathrm{ng} / \mathrm{ml}$. Total plasma Hcy was determined by an enzymatic assay on a Hitachi 917 analyser (Roche Diagnostics), using reagents and calibrators from Catch Inc. (Seattle, WA, USA). The CV was $2 \cdot 1 \%$ to $5 \cdot 3 \%$.

Maternal plasma ascorbic acid concentrations were determined by HPLC with metaphosphoric acid-stabilized plasma using previously described methods ${ }^{(37)}$. All samples were analysed in duplicate and in a blinded fashion. The intra- and inter-assay CV was 3.5\% and 5.5\%, respectively. The quantification of serum carotenoids, retinol and $\alpha$-tocopherol was by HPLC using a method based on that described by Browne and Armstrong ${ }^{(38)}$. The inter-assay $\mathrm{CV}$ for each lipid-soluble carotenoid/vitamin was $12 \%$ for lutein and zeaxanthin (determined as a single peak), $11 \%$ for $\beta$-cryptoxanthin, $9 \%$ for lycopene, $11 \%$ for $\beta$-carotene, $8 \%$ for retinol and $6 \%$ for $\alpha$-tocopherol. $\alpha$-Tocopherol concentrations were lipid-standardized by dividing by total cholesterol concentrations, which were determined enzymatically using specific reagents from Pointe Scientific (Canton, MI, USA).

Vitamin D status was assessed using 25-hydroxyvitamin D (25(OH)D), which was quantified using a commercial ELISA from Immunodiagnostic Systems Limited (Bolden, UK) and validated against an HPLC method as described 
previously ${ }^{(39)}$. Serum ferritin was analysed using an immunoradiometric assay with ${ }^{125}$ I-labelled anti-ferritin antibody in a kit obtained from Diagnostic Products Corporation (Los Angeles, CA, USA). The inter-assay CV was $16 \cdot 4 \%$. Serum soluble transferrin receptors were measured using an ELISA from R\&D Systems (Minneapolis, MN, USA). The inter-assay CV ranged from 8.5 to $17 \%$. All samples were analysed in duplicate and in a blinded fashion.

A semi-quantitative modified Block98 FFQ was selfadministered at the 30-week visit to inquire about usual dietary intake in the past 3 months; the instrument has been validated in many populations, including pregnant women ${ }^{(40,41)}$. The Block98 FFQ assesses fifty-one nutrients and seven food groups from approximately 120 food/beverage items and was modified to focus on a 3-month time period ${ }^{(42)}$. Completed questionnaires were sent to Block Dietary Data Systems (Berkeley, CA, USA) for optical scanning and nutrient analysis using software developed at the National Cancer Institute.

Mothers self-reported their race/ethnicity (non-Hispanic white, non-Hispanic black, other), age, parity $(0, \geq 1)$, smoking status (smoker, non-smoker), education (less than high school, high school or equivalent, some college), marital status (married, unmarried) and work status (full-time, part-time or none). Pre-pregnancy BMI (weight $(\mathrm{kg}) /[\text { height }(\mathrm{m})]^{2}$ ) was based on maternal recall of prepregnancy weight at enrolment and measured height. BMI categories were underweight $\left(\mathrm{BMI}<18.5 \mathrm{~kg} / \mathrm{m}^{2}\right)$, normal weight $\left(\mathrm{BMI}=18 \cdot 5-24.9 \mathrm{~kg} / \mathrm{m}^{2}\right)$, overweight $\left(\mathrm{BMI}=25 \cdot 0-29 \cdot 9 \mathrm{~kg} / \mathrm{m}^{2}\right)$ and obese $\left(\mathrm{BMI} \geq 30 \cdot 0 \mathrm{~kg} / \mathrm{m}^{2}\right)^{(43)}$. Gestational age was determined by maternal report of the date of the last menstrual period and was confirmed with ultrasound when available. Self-reported use of selective serotonin reuptake inhibitors was verified using serum concentrations.

\section{Statistical analysis}

Many of the biomarkers exhibited skewed distributions, thus violating the assumption of normality required by factor analytic procedures. We therefore factor analysed a matrix constructed of Spearman's $\rho$ correlation coefficients. Factors were extracted from a principal components analysis and orthogonally rotated with the varimax method. Solutions for two to eight factors were examined. The number of factors extracted was based on examination of eigenvalues, scree plots and the interpretability of the solution. The estimated factor scores were computed using a regression method ${ }^{(44)}$ based on fourteen biomarkers. Serum ferritin was initially included in the analysis, but dropped before the final factors were extracted because it did not load highly on any of the final factors. All factor loadings $\geq|0 \cdot 3|$ were presented. Factor scores were then categorized based on tertiles of the distribution.

We used a non-parametric test of trend to determine differences in maternal characteristics and nutritional biomarker concentrations across tertiles of each factor score. Spearman correlation coefficients were calculated between factor scores and measures of dietary intake. We used a $\chi^{2}$ test to determine if the prevalence of MDD varied by study visit. To test for an association between factor scores at 20 weeks' gestation and MDD, depression status at each time point during pregnancy was used as the dependent measure in a longitudinal logistic regression model employing generalized estimating equations, which account for intra-individual correlation of depression measurements. Gestational age at the study visit was included in all models. Other potential confounding variables (race/ethnicity, pre-pregnancy BMI, maternal age, education, marital status, work status, parity, antidepressant use and season) were included in the final model if their inclusion resulted in a change in the main-effect odds ratio of $\geq 10 \%{ }^{(45)}$. Pre-pregnancy BMI, education and work status met this definition. We tested for interaction between factor scores and study visit using a Wald $P$ value $(P<0 \cdot 05)$, but because no interaction was observed, models were not stratified by study visit. Our sample size gave us $80 \%$ power to find a medium effect size $(f=0 \cdot 31)$. Analyses were conducted using the SATA statistical software package release 10 (StataCorp LP, College Station, TX, USA).

\section{Results}

Most women in the cohort were white, 25-34 years old, college-educated, married, had one or more previous live births and had a pre-pregnancy BMI $\geq 25 \cdot 0 \mathrm{~kg} / \mathrm{m}^{2}$ (Table 1 ). Mean concentrations of each nutritional biomarker at $\leq 20$ weeks' gestation included in the factor analysis indicate that this population was well-nourished. We derived three patterns using the biomarker data (Table 2). Factor 1 (Essential Fatty Acids) had high factor loadings on red cell DHA, AA and EPA. Plasma folate, plasma ascorbic acid, serum retinol, serum 25(OH)D, serum $\alpha$-tocopherol and serum $\beta$-carotene loaded positively, and plasma Hcy and serum soluble transferrin receptors loaded negatively on Factor 2 (Micronutrients). Factor 3 (Carotenoids) had high factor loadings for serum carotenoids. Before rotation, these factors explained $46 \%$ of the variance in biomarker concentrations.

Women in the highest tertile of all three biomarker patterns (Essential Fatty Acids, Micronutrients and Carotenoids) were more likely than women in the lower tertiles to be primiparous and lean (Table 3). Well-educated women were more often in the upper tertiles of the Micronutrients and Carotenoids patterns. As Micronutrients factor tertiles increased, a greater proportion of women were white, older and married.

In the subset of the sample with dietary data ( $n$ 107), Essential Fatty Acids scores had weak, negative correlations with intakes of energy, red meat, poultry, processed meats and fried foods and a weak positive correlation 
Table 1 Characteristics of women in the cohort $(n$ 135), Antidepressant Use During Pregnancy Study, Pittsburgh, Pennsylvania, USA

\begin{tabular}{|c|c|c|}
\hline & $\%$ or Geometric mean & $95 \% \mathrm{Cl}$ \\
\hline \multicolumn{3}{|l|}{ Maternal race (\%) } \\
\hline White & 81 & \\
\hline Black & 19 & \\
\hline \multicolumn{3}{|l|}{ Maternal age (years; \%) } \\
\hline $18-24$ & 13 & \\
\hline $25-34$ & 64 & \\
\hline $35-43$ & 23 & \\
\hline \multicolumn{3}{|l|}{ Education (\%) } \\
\hline High school or less & 18 & \\
\hline Some college & 19 & \\
\hline College graduate & 36 & \\
\hline Some graduate school & 27 & \\
\hline Full-time work or school (\%) & 31 & \\
\hline Married (\%) & 74 & \\
\hline Currently nulliparous (\%) & 32 & \\
\hline Current smoker (\%) & 16 & \\
\hline \multicolumn{3}{|l|}{ Pre-pregnancy BMI (kg/m²; \%) } \\
\hline$<25 \cdot 0$ & 49 & \\
\hline $25 \cdot 0-29 \cdot 9$ & 23 & \\
\hline$\geq 30 \cdot 0$ & 28 & \\
\hline Red-cell DHA (\% weight) & $2 \cdot 80$ & $2 \cdot 36,3 \cdot 33$ \\
\hline Red-cell arachidonic acid (\% weight) & $11 \cdot 8$ & $10 \cdot 7,13 \cdot 1$ \\
\hline Red-cell EPA (\% weight) & $0 \cdot 30$ & $0.26,0.34$ \\
\hline Plasma folate $(\mathrm{nmol} / \mathrm{l})$ & $31 \cdot 5$ & $28 \cdot 9,34 \cdot 3$ \\
\hline Plasma ascorbic acid (nmol/l) & $66 \cdot 1$ & $61 \cdot 6,70 \cdot 8$ \\
\hline Serum retinol $(\mathrm{nmol} / \mathrm{l})$ & 1649 & 1580,1721 \\
\hline Serum 25-hydroxyvitamin D (nmol/l) & $81 \cdot 9$ & $75 \cdot 1,89 \cdot 3$ \\
\hline Serum $\alpha$-tocopherol (nmol/l) & 161 & 156,165 \\
\hline Serum $\beta$-carotene $(\mathrm{nmol} / \mathrm{l})$ & 162 & 127,206 \\
\hline Plasma homocysteine ( $\mu \mathrm{mol} / \mathrm{l})$ & $2 \cdot 32$ & $2 \cdot 17,2 \cdot 48$ \\
\hline Serum soluble transferrin receptors $(\mu \mathrm{mol} / \mathrm{l})$ & $15 \cdot 2$ & $14 \cdot 5,15 \cdot 9$ \\
\hline Serum lutein + zeaxanthin $(\mathrm{nmol} / \mathrm{l})$ & 102 & $90 \cdot 5,114$ \\
\hline Serum $\beta$-cryptoxanthin (nmol/l) & 132 & 110,158 \\
\hline Serum lycopene $(\mathrm{nmol} / \mathrm{l})$ & 541 & 489,598 \\
\hline
\end{tabular}

Table 2 Rotated factor loadings for maternal dietary biomarker concentrations ( $n$ 135)t, Antidepressant Use During Pregnancy Study, Pittsburgh, Pennsylvania, USA

\begin{tabular}{lccc}
\hline & Essential Fatty Acids & Micronutrients & Carotenoids \\
\hline Red-cell DHA & 0.94 & - & - \\
Red-cell arachidonic acid & 0.87 & - & - \\
Red-cell EPA & 0.66 & - & - \\
Plasma folate & - & 0.55 & - \\
Plasma ascorbic acid & - & 0.55 & - \\
Serum retinol & - & 0.51 & - \\
Serum 25-hydroxyvitamin D & - & 0.47 & - \\
Serum $\alpha$-tocopherol & - & 0.32 & 0.68 \\
Serum $\beta$-carotene & - & 0.30 & - \\
Plasma homocysteine & - & -0.58 & - \\
Serum soluble transferrin receptors & - & -0.56 & 0.77 \\
Serum lutein + zeaxanthin & - & - & 0.66 \\
Serum $\beta$-cryptoxanthin & - & - & 0.52 \\
Serum lycopene & - & - &
\end{tabular}

†Factor analysis on fourteen biomarkers. Only factor loadings $\geq|0 \cdot 3|$ are presented.

with soya-based food intakes. However, scores were not correlated with fish and seafood intakes (Table 4). Micronutrients factor scores had weak, positive correlations with intakes of fruits, vegetables, whole grains, reduced-fat dairy products, nuts and soya-based foods, and weak negative correlations with intakes of energy, high-fat dairy products, processed meat, fried foods and sugar-sweetened beverages.
Intakes of fruits, vegetables, legumes, nuts and soya-based foods had weak positive correlations, and intakes of energy, high-fat dairy products, processed meat, fried foods and sugar-sweetened beverages had weak negative correlations with Carotenoids factor scores.

MDD occurred in $21.5 \%$ of women overall and did not vary by study visit $(P=0 \cdot 8)$. The prevalence of MDD was not 
Table 3 Maternal characteristics in the lowest (T1) and highest (T3) tertile of biomarker factor scores ( $n$ 135)t, Antidepressant Use During Pregnancy Study, Pittsburgh, Pennsylvania, USA

\begin{tabular}{|c|c|c|c|c|c|c|}
\hline & \multicolumn{2}{|c|}{ Essential Fatty Acids } & \multicolumn{2}{|c|}{ Micronutrients } & \multicolumn{2}{|c|}{ Carotenoids } \\
\hline & T1 (low) & T3 (high) & T1 (low) & T3 (high) & T1 (low) & T3 (high) \\
\hline \multicolumn{7}{|l|}{ Maternal race (\%) } \\
\hline White & 84 & 78 & 56 & $100^{\star *}$ & 80 & 86 \\
\hline Black & 16 & 22 & 44 & 0 & 20 & 14 \\
\hline \multicolumn{7}{|l|}{ Maternal age (years; \%) } \\
\hline $18-24$ & 11 & 13 & 29 & $5^{\star \star}$ & 20 & 9 \\
\hline $25-34$ & 62 & 62 & 60 & 68 & 69 & 62 \\
\hline $35-43$ & 27 & 24 & 11 & 27 & 11 & 29 \\
\hline \multicolumn{7}{|l|}{ Education (\%) } \\
\hline High school or less & 22 & 9 & 31 & $4^{\star \star}$ & 27 & $9^{\star \star}$ \\
\hline Some college & 18 & 24 & 33 & 16 & 33 & 9 \\
\hline College graduate & 31 & 40 & 20 & 49 & 20 & 47 \\
\hline Some graduate school & 29 & 27 & 16 & 31 & 20 & 35 \\
\hline \multicolumn{7}{|l|}{ Work status (\%) } \\
\hline Part-time or none & 73 & 62 & 60 & 64 & 58 & 76 \\
\hline Full-time work/school & 27 & 38 & 40 & 36 & 42 & 24 \\
\hline \multicolumn{7}{|l|}{ Marital status (\%) } \\
\hline Married & 78 & 76 & 56 & $91^{* *}$ & 69 & 82 \\
\hline Unmarried & 22 & 24 & 44 & 9 & 31 & 18 \\
\hline \multicolumn{7}{|l|}{ Current parity (\%) } \\
\hline 0 & 24 & $49^{*}$ & 16 & $42^{*}$ & 24 & $51^{\star \star}$ \\
\hline 1 or more & 76 & 51 & 84 & 58 & 76 & 49 \\
\hline \multicolumn{7}{|l|}{ Current smoking status (\%) } \\
\hline Smoker & 16 & 9 & 16 & 7 & 20 & 7 \\
\hline Non-smoker & 84 & 91 & 84 & 93 & 80 & 93 \\
\hline \multicolumn{7}{|c|}{ Pre-pregnancy BMI $\left(\mathrm{kg} / \mathrm{m}^{2} ; \%\right)$} \\
\hline$<25 \cdot 0$ & 36 & $69^{\star *}$ & 31 & $69^{\star \star}$ & 30 & $76^{\star \star}$ \\
\hline $25 \cdot 0-29 \cdot 9$ & 23 & 13 & 27 & 20 & 20 & 18 \\
\hline$\geq 30 \cdot 0$ & 41 & 18 & 42 & 11 & 50 & 7 \\
\hline
\end{tabular}

Distribution of characteristics was significantly different across tertiles: ${ }^{\star} P<0 \cdot 05,{ }^{* *} P<0 \cdot 01$.

†Tertile 2 was omitted for simplicity.

Table 4 Correlation between factor scores and selected measures of dietary intake ( $n$ 107)t, Antidepressant Use During Pregnancy Study, Pittsburgh, Pennsylvania, USA

\begin{tabular}{|c|c|c|c|}
\hline & Essential Fatty Acids & Micronutrients & Carotenoids \\
\hline Energy & $-0 \cdot 24^{\star}$ & $-0.22^{*}$ & $-0 \cdot 25^{\star \star}$ \\
\hline Fruits & $0 \cdot 10$ & $0 \cdot 19^{*}$ & $0 \cdot 24^{\star}$ \\
\hline Vegetables & 0.03 & $0 \cdot 32^{\star \star}$ & $0 \cdot 31^{\star *}$ \\
\hline Whole grains & 0.07 & $0 \cdot 23^{*}$ & $0 \cdot 17$ \\
\hline High-fat dairy products & -0.06 & $-0 \cdot 27^{\star \star}$ & $-0.22^{*}$ \\
\hline Reduced-fat dairy products & 0.04 & $0 \cdot 33^{\star \star}$ & $0 \cdot 18$ \\
\hline Legumes & $0 \cdot 10$ & 0.06 & $0 \cdot 32^{\star \star}$ \\
\hline Nuts & $0 \cdot 11$ & $0 \cdot 23^{*}$ & $0 \cdot 30^{\star \star}$ \\
\hline Soya-based foods & $0 \cdot 24^{*}$ & $0 \cdot 39^{\star \star}$ & $0 \cdot 39^{\star \star}$ \\
\hline Red meat & $-0 \cdot 22^{*}$ & $-0 \cdot 16$ & $-0 \cdot 19$ \\
\hline Poultry, not fried & $-0.22^{\star}$ & -0.09 & 0.01 \\
\hline Fish and seafood & 0.08 & -0.02 & 0.04 \\
\hline Processed meat & $-0 \cdot 27^{\star \star}$ & $-0 \cdot 33^{\star \star}$ & $-0 \cdot 31^{\star \star}$ \\
\hline Fried foods & $-0 \cdot 20^{\star}$ & $-0 \cdot 44^{\star \star}$ & $-0 \cdot 38^{\star *}$ \\
\hline Sugar-sweetened beverages & $-0 \cdot 14$ & $-0 \cdot 35^{\star \star}$ & $-0 \cdot 45^{\star \star}$ \\
\hline
\end{tabular}

Correlation was statistically significant: ${ }^{\star} P<0.05,{ }^{\star \star} P<0.01$. tValues in the table are correlation coefficients.

significantly different across tertiles of any factor scores at $\leq 20$ weeks' gestation (Table 5). In longitudinal models, there was no association between Essential Fatty Acids scores or Micronutrients scores and MDD either before or after confounder adjustment. Women with Carotenoids factor scores in the middle and upper tertiles were $60 \%$ less likely than women in the bottom tertile to have MDD during pregnancy, but after adjustment for pre-pregnancy BMI, employment and education status, associations were no longer statistically significant. Alternative specifications of the factor scores (e.g. as a continuous variable, spline variables or as different categories) did not change these conclusions. 
Table 5 Association between dietary biomarker factor scores at $<20$ weeks' gestation and major depressive disorder during pregnancy (n 135 women contributing 352 person-observations), Antidepressant Use During Pregnancy Study, Pittsburgh, Pennsylvania, USA

\begin{tabular}{|c|c|c|c|c|c|}
\hline \multirow[b]{2}{*}{ Biomarker factor } & \multirow{2}{*}{$\begin{array}{l}\text { Unadjusted prevalence of major } \\
\text { depressive disorder at enrolment }\end{array}$} & \multicolumn{2}{|c|}{ Model 1† } & \multicolumn{2}{|c|}{ Model 2‡ } \\
\hline & & OR & $95 \% \mathrm{Cl}$ & OR & $95 \% \mathrm{Cl}$ \\
\hline \multicolumn{6}{|l|}{ Essential Fatty Acids } \\
\hline Tertile 1 (lowest) & $26 \cdot 7$ & $1 \cdot 0$ & Ref. & $1 \cdot 0$ & Ref. \\
\hline Tertile 2 & $26 \cdot 7$ & $1 \cdot 0$ & $0 \cdot 4,2 \cdot 4$ & $1 \cdot 1$ & $0 \cdot 4,2 \cdot 6$ \\
\hline Tertile 3 (highest) & $20 \cdot 0$ & 0.9 & $0 \cdot 4,2 \cdot 0$ & 0.8 & $0 \cdot 3,2 \cdot 1$ \\
\hline \multicolumn{6}{|l|}{ Micronutrients } \\
\hline Tertile 1 (lowest) & $31 \cdot 1$ & $1 \cdot 0$ & Ref. & $1 \cdot 0$ & Ref. \\
\hline Tertile 2 & $17 \cdot 8$ & 0.6 & $0 \cdot 3,1 \cdot 4$ & $1 \cdot 1$ & $0.4,3 \cdot 0$ \\
\hline Tertile 3 (highest) & $24 \cdot 4$ & 0.7 & $0 \cdot 3,1 \cdot 7$ & $1 \cdot 4$ & $0 \cdot 5,3 \cdot 8$ \\
\hline \multicolumn{6}{|l|}{ Carotenoids } \\
\hline Tertile 1 (lowest) & $35 \cdot 6$ & $1 \cdot 0$ & Ref. & $1 \cdot 0$ & Ref. \\
\hline Tertile 2 & $20 \cdot 0$ & 0.4 & $0.2,0.9$ & 0.5 & $0 \cdot 2,1 \cdot 4$ \\
\hline Tertile 3 (highest) & $17 \cdot 7$ & 0.4 & $0.2,0.9$ & 0.8 & $0 \cdot 3,2 \cdot 1$ \\
\hline
\end{tabular}

Ref., referent category.

tAdjusted for study visit number.

$\ddagger$ Adjusted for study visit number, employment, education and pre-pregnancy BMI. Additional adjustment for other covariates had no meaningful impact on the findings.

\section{Discussion}

In this cohort of pregnant women we used factor analysis to derive three patterns in nutritional biomarkers that we labelled Essential Fatty Acids, Micronutrients and Carotenoids. We used factor analysis rather than other data reduction techniques because we believe that maternal nutritional biomarker concentrations are a manifestation of latent underlying constructs (factors) that may be reflective of meaningful dietary patterns in pregnancy. The study of patterns using dietary intake data is widespread in nutritional epidemiology ${ }^{(46-50)}$, and in other fields, biochemical markers of metabolism, inflammation and antioxidant status have commonly been studied using pattern analysis ${ }^{(51-55)}$. Nevertheless, we are aware of only one other study that identified patterns using nutritional biomarker concentrations. In a sample of 310 lactating women from The Netherlands, Mueller et al. ${ }^{(56)}$ derived two major patterns of fatty acid concentrations in maternal breast milk samples using principal component analysis: (i) 18:1 trans-isomers; and (ii) markers of dairy fat including 15:0, 17:0, trans 11-18:1 and cis 9, trans 11-18:2. Like foods and nutrients measured from self-reported dietary instruments, nutritional biomarkers can also be highly correlated. Thus, creating dietary patterns using biomarker data seems a reasonable approach to examining dietary intakes, and has the distinct advantage of employing objective measures of intake. Biomarker patterns may also be more meaningful than a single nutritional biomarker since resultant patterns capture synergistic interactions among nutrients.

Our study is unique in that we identified patterns using nutritional biomarkers of a wide range of nutrients that are important during pregnancy. Notably, the three factors we identified using maternal biomarkers represented patterns reflective of expected food intakes while also capturing additional differences in food intakes across patterns. For instance, Micronutrients scores were positively correlated with many foods that contribute to folate, $\beta$-carotene and vitamins C, A, E and D (nutrients that loaded highly on this factor), including fruits, vegetables, whole grains, reduced-fat dairy products, nuts and soya products. However, scores were also associated with foods and nutrients not specifically represented in the biomarkers, such as reduced intakes of sugar-sweetened beverages, fried foods and processed meat. Likewise, Carotenoids scores were correlated with fruits and vegetables (the primary sources of carotenoids), but also with intake of other healthy foods. Surprisingly, Essential Fatty Acids scores were not associated with fish and seafood intakes, but were associated with intakes of soya-based foods (positively) and energy, red meat, poultry, processed meats and fried foods (negatively). It is possible that the Block98 FFQ used in the present study may not have adequately captured adequate variation in fish and seafood intakes based on its five line items. Moreover, it is likely that in this well-educated cohort of women interested in participating in a study on depression, fish oil use was more common than in other populations thus leading to this major pattern we observed. Unfortunately, we lacked data on dietary supplement use (such as fish oil) to better understand how dietary supplement use impacted our biomarker patterns.

In our cohort, we found no independent association between Essential Fatty Acids, Micronutrients or Carotenoids scores in mid-pregnancy and the prevalence of MDD at 20, 30 and 36 weeks. It is difficult to compare our results with others because of differences in study design, nutrition assessment methods, definitions of depression, confounders considered and characteristics of the population. Importantly, little work has explored nutrition and depression in the context of pregnancy ${ }^{(20-25)}$, when unique stresses and physiological adaptation to gestation may alter associations with mental health outcomes.

Nutrients that we included in our biomarker patterns have been implicated in $\mathrm{MDD}^{(17,20,27)}$. Each nutrient we 
studied has a recognized role in brain functioning, and some degree of research supporting an association with $\operatorname{mood}^{(17-20,27)}$. Nevertheless, evidence is not conclusive. For instance, $n-3$ (omega-3) fatty acid supplements may help to improve depressive symptoms, but only among individuals diagnosed with depressive illness ${ }^{(13)}$. Poor folate status has been related to depression in predominantly cross-sectional and case-control studies ${ }^{(15)}$, but few randomized trials and rigorous prospective studies have explored the causal and temporal natures of this relationship ${ }^{(57)}$. Similarly, research into the role of vitamin D in depression is mixed, with most studies suffering from important methodological limitations ${ }^{(9)}$.

While we observed meaningful dietary patterns in our study using objective data based on nutritional biomarkers, our lack of an association between the patterns and depression at 20, 30 and 36 weeks may be due to several factors. First, given the high socio-economic status of the sample, use of prenatal vitamins and other dietary supplements may have been widespread, and our lack of supplement use data prevents us from quantifying use directly and observing its impact on the association. Likewise, it is possible that with a predominance of wellnourished women, associations between poor nutrition and antenatal MDD could not be observed. Our relatively small sample of mostly well-educated, white women with a high prevalence of depression may not generalize to the majority of pregnant women in the USA. Indeed the depressed women in our sample, who were functional enough to participate in this research study while pregnant, may be less likely to have nutritional deficiencies as a significant contributor to MDD, compared with less functional women. Our results may also have been different if we had included women with other psychiatric illnesses that have a greater impact than depression on appetite and metabolism, such as bipolar disorder and schizophrenia. Exploring these disorders in pregnant women may reveal different relationships between nutritional status, dietary intake and psychiatric symptoms. Finally, we were powered to detect a medium-sized effect. If the effect size between nutritional patterns and depression is small, we could not detect this difference.

Strengths of our study included the longitudinal nature of the data and use of the gold-standard assessment of MDD based on clinical diagnosis. Our use of a full panel of nutritional biomarkers (objective measures of dietary intakes) allowed us to overcome the limitations of selfreported diet. As well, factor analysis has not been often used to characterize dietary patterns using biomarker data, and we observed three interesting patterns using this approach. Further, factor analysis allowed us to account for the correlated nature of dietary biomarkers and provided a novel assessment of maternal nutritional status mid-pregnancy.

In conclusion, dietary patterns using nutritional biomarkers among women in mid-pregnancy were not independently associated with antenatal MDD in the present study. Future studies in diverse cohorts of pregnant women with rigorous measures are needed to clarify the temporality, direction and magnitude of the association between nutrition and depression in pregnancy. Because nutritional status is a modifiable exposure, determining its contribution to depression during pregnancy is of major public health significance.

\section{Acknowledgements}

This work was supported by the National Institutes of Health grants K01 MH074092 (Principal Investigator: L.M.B.) and R01 MH060335 (Principal Investigator: K.L.W.). None of the authors had personal or financial conflict of interests. L.M.B, K.L.W. and P.K.N. designed the research; K.L.W. provided the data; R.W.P., R.W.E. and M.J.G. generated biomarker assay data for the study; L.M.B. and J.F.L. analysed data; L.M.B., K.L.W. and P.K.N. wrote the manuscript; J.F.L., R.W.P. and R.W.E. provided significant advice and critically edited the manuscript; all authors read and approved the final manuscript.

\section{References}

1. Kessler RC (2003) Epidemiology of women and depression. $J$ Affect Disord 74, 5-13.

2. Gaynes BN, Gavin N, Meltzer-Brody S et al. (2005) Perinatal Depression: Prevalence, Screening Accuracy, and Screening Outcomes. Evidence Report/Technology Assessment no. 119 (Prepared by the RTI-University of North Carolina Evidence-based Practice Center, under Contract No. 290-02-0016). AHRQ Publication no. 05E006-2. Rockville, MD: Agency for Healthcare Research and Quality.

3. Yonkers KA, Wisner KL, Stewart DE et al. (2009) The management of depression during pregnancy: a report from the American Psychiatric Association and the American College of Obstetricians and Gynecologists. Obstet Gynecol 114, 703-713.

4. Alder J, Fink N, Bitzer J et al. (2007) Depression and anxiety during pregnancy: a risk factor for obstetric, fetal and neonatal outcome? A critical review of the literature. J Matern Fetal Neonatal Med 20, 189-209.

5. Grote NK, Bridge JA, Gavin AR et al. (2010) A meta-analysis of depression during pregnancy and the risk of preterm birth, low birth weight, and intrauterine growth restriction. Arch Gen Psychiatry 67, 1012-1024.

6. Zuckerman B, Bauchner H, Parker S et al. (1990) Maternal depressive symptoms during pregnancy, and newborn irritability. J Dev Behav Pediatr 11, 190-194.

7. Hirschfeld RM, Keller MB, Panico S et al. (1997) The National Depressive and Manic-Depressive Association consensus statement on the undertreatment of depression. JAMA 277, 333-340.

8. Benton D \& Cook R (1991) The impact of selenium supplementation on mood. Biol Psychiatry 29, 1092-1098.

9. Bertone-Johnson ER (2009) Vitamin D and the occurrence of depression: causal association or circumstantial evidence? Nutr Rev 67, 481-492.

10. Murakami K \& Sasaki S (2010) Dietary intake and depressive symptoms: a systematic review of observational studies. Mol Nutr Food Res 54, 471-488. 
11. Jacka FN, Pasco JA, Mykletun A et al. (2010) Association of Western and traditional diets with depression and anxiety in women. Am J Psychiatry 167, 305-311.

12. Kuczmarski MF, Cremer Sees A, Hotchkiss L et al. (2010) Higher Healthy Eating Index-2005 scores associated with reduced symptoms of depression in an urban population: findings from the Healthy Aging in Neighborhoods of Diversity Across the Life Span (HANDLS) study. J Am Diet Assoc 110, 383-389.

13. Appleton KM, Rogers PJ \& Ness AR (2010) Updated systematic review and meta-analysis of the effects of $n-3$ long-chain polyunsaturated fatty acids on depressed mood. Am J Clin Nutr 91, 757-770.

14. Assies J, Pouwer F, Lok A et al. (2010) Plasma and erythrocyte fatty acid patterns in patients with recurrent depression: a matched case-control study. PLoS One 5, e10635.

15. Gilbody S, Lightfoot $\mathrm{T}$ \& Sheldon $\mathrm{T}$ (2007) Is low folate a risk factor for depression? A meta-analysis and exploration of heterogeneity. I Epidemiol Community Health 61, 631-637.

16. Brody S (2002) High-dose ascorbic acid increases intercourse frequency and improves mood: a randomized controlled clinical trial. Biol Psychiatry 52, 371-374.

17. Benton D \& Donohoe RT (1999) The effects of nutrients on mood. Public Health Nutr 2, 403-409.

18. Benton D, Haller J \& Fordy J (1995) Vitamin supplementation for 1 year improves mood. Neuropsychobiology 32, 98-105.

19. Rucklidge JJ, Gately D \& Kaplan BJ (2010) Database analysis of children and adolescents with bipolar disorder consuming a micronutrient formula. BMC Psychiatry 10, 74.

20. Bodnar LM \& Wisner KL (2005) Nutrition and depression: implications for improving mental health among childbearing-aged women. Biol Psychiatry 58, 679-685.

21. Hurley KM, Caulfield LE, Sacco LM et al. (2005) Psychosocial influences in dietary patterns during pregnancy. J Am Diet Assoc 105, 963-966.

22. Strom M, Mortensen EL, Halldorsson TI et al. (2009) Fish and long-chain $n-3$ polyunsaturated fatty acid intakes during pregnancy and risk of postpartum depression: a prospective study based on a large national birth cohort. Am J Clin Nutr 90, 149-155.

23. Leung BM \& Kaplan BJ (2009) Perinatal depression: prevalence, risks, and the nutrition link - a review of the literature. J Am Diet Assoc 109, 1566-1575.

24. Golding J, Steer C, Emmett P et al. (2009) High levels of depressive symptoms in pregnancy with low omega-3 fatty acid intake from fish. Epidemiology 20, 598-603.

25. Otto SJ, de Groot RH \& Hornstra G (2003) Increased risk of postpartum depressive symptoms is associated with slower normalization after pregnancy of the functional docosahexaenoic acid status. Prostaglandins Leukot Essent Fatty Acids 69, 237-243.

26. Institute of Medicine (2006) Dietary Reference Intakes: The Essential Guide to Nutrient Requirements. Washington, DC: National Academies Press.

27. Prasad C (2001) Improving mental health through nutrition: the future. Nutr Neurosci 4, 251-272.

28. Hunter D (1998) Biochemical indicators of dietary intake. In Nutritional Epidemiology, 2nd ed., pp. 174-243 [WC Willett, editor]. New York: Oxford University Press.

29. Wisner KL, Sit DK, Hanusa BH et al. (2009) Major depression and antidepressant treatment: impact on pregnancy and neonatal outcomes. Am J Psychiatry 166, 557-566.

30. First MB, Spitzer RL, Gibbon MW et al. (1994) Structured Clinical Interview for DSM-IV Axis II Personality Disorders (SCID-II), Version 2.O. New York: Biometrics Research Department, New York State Psychiatric Institute.
31. Post RM, Roy-Byrne PP \& Uhde TW (1988) Graphic representation of the life course of illness in patients with affective disorder. Am J Psychiatry 145, 844-848.

32. Wisner KL, Peindl KS \& Hanusa BH (1995) Psychiatric episodes in women with young children. J Affect Disord 34, 1-11.

33. Williams J \& Terman M (2003) Structured Interview Guide for the Hamilton Depression Rating Scale with Atypical Depression Supplement (SIGH-ADS). New York: New York State Psychiatric Institute.

34. Hamilton M (1960) A rating scale for depression. J Neurol Neurosurg Psychiatry 23, 56-62.

35. Bligh EG \& Dyer WJ (1959) A rapid method of total lipid extraction and purification. Can J Biochem Physiol 37, 911-917.

36. Morrison WR \& Smith LM (1964) Preparation of fatty acid methyl esters and dimethylacetals from lipids with boron fluoride-methanol. J Lipid Res 5, 600-608.

37. Rumelin A, Fauth U \& Halmagyi M (1999) Determination of ascorbic acid in plasma and urine by high performance liquid chromatography with ultraviolet detection. Clin Chem Lab Med 37, 533-536.

38. Browne RW \& Armstrong D (1998) Simultaneous determination of serum retinol, tocopherols, and carotenoids by HPLC. Methods Mol Biol 108, 269-275.

39. Bodnar LM, Simhan HN, Powers RW et al. (2007) High prevalence of vitamin D insufficiency in black and white pregnant women residing in the northern United States and their neonates. J Nutr 137, 447-452.

40. Block G, Thompson FE, Hartman AM et al. (1992) Comparison of two dietary questionnaires validated against multiple dietary records collected during a 1-year period. J Am Diet Assoc 92, 686-693.

41. Block G, Woods M, Potosky A et al. (1990) Validation of a self-administered diet history questionnaire using multiple diet records. J Clin Epidemiol 43, 1327-1335.

42. Block G, Hartman AM, Dresser CM et al. (1986) A databased approach to diet questionnaire design and testing. Am J Epidemiol 124, 453-469.

43. World Health Organization (2000) Obesity: Preventing and Managing the Global Epidemic. WHO Technical Report Series no. 894. Geneva: WHO.

44. Grice JW (2001) A comparison of factor scores under conditions of factor obliquity. Psychol Methods 6, 67-83.

45. Weng HY, Hsueh YH, Messam LL et al. (2009) Methods of covariate selection: directed acyclic graphs and the changein-estimate procedure. Am J Epidemiol 169, 1182-1190.

46. Newby PK \& Tucker KL (2004) Empirically derived eating patterns using factor or cluster analysis: a review. Nutr Rev 62, 177-203.

47. Moeller SM, Reedy J, Millen AE et al. (2007) Dietary patterns: challenges and opportunities in dietary patterns research an Experimental Biology workshop, April 1, 2006. J Am Diet Assoc 107, 1233-1239.

48. Hu FB (2002) Dietary pattern analysis: a new direction in nutritional epidemiology. Curr Opin Lipidol 13, 3-9.

49. Tucker KL (2010) Dietary patterns, approaches, and multicultural perspective. Appl Physiol Nutr Metab 35, 211-218

50. Kant AK (2010) Dietary patterns: biomarkers and chronic disease risk. Appl Physiol Nutr Metab 35, 199-206.

51. Dewailly D, Pigny P, Soudan B et al. (2010) Reconciling the definitions of polycystic ovary syndrome: the ovarian follicle number and serum anti-Mullerian hormone concentrations aggregate with the markers of hyperandrogenism. J Clin Endocrinol Metab 95, 4399-4405.

52. Shah SH, Bain JR, Muehlbauer MJ et al. (2010) Association of a peripheral blood metabolic profile with coronary artery disease and risk of subsequent cardiovascular events. Circ Cardiovasc Genet 3, 207-214. 
53. van den Ham HJ, de Jager W, Bijlsma JW et al. (2009) Differential cytokine profiles in juvenile idiopathic arthritis subtypes revealed by cluster analysis. Rheumatology (Oxford) 48, 899-905.

54. Aronson D, Avizohar O, Levy Y et al. (2008) Factor analysis of risk variables associated with low-grade inflammation. Atherosclerosis 200, 206-212.

55. Botelho PB, Fioratti CO, Abdalla DS et al. (2010) Classification of individuals with dyslipidaemia controlled by statins according to plasma biomarkers of oxidative stress using cluster analysis. Br J Nutr 103, 256-265.

56. Mueller A, Thijs C, Rist L et al. (2010) Trans fatty acids in human milk are an indicator of different maternal dietary sources containing trans fatty acids. Lipids 45, 245-251.

57. Walker JG, Mackinnon AJ, Batterham P et al. (2010) Mental health literacy, folic acid and vitamin $\mathrm{B}_{12}$, and physical activity for the prevention of depression in older adults: randomised controlled trial. Br J Psychiatry 197, 45-54. 